BNL - 68351

\title{
Inelastic X-Ray Scattering at Ultrahigh Pressures
}

\author{
Ho-kwang Mao and Russell J. Hemley \\ Geophysical Laboratory and HPCAT \\ Carnegie Institution \\ 5251 Broad Branch Road \\ NW, Washington, DC 20015-1305 \\ Chi-Chang Kao \\ National Synchrotron Light Source \\ Brookhaven National Laboratory \\ P.O. Box 5000 \\ Upton, NY, USA 11973-5000
}

September 2000

\section{National Synchrotron Light Source}

\author{
Brookhaven National Laboratory \\ Operated by \\ Brookhaven Science Associates \\ Upton, NY 11973
}

Under Contract with the United States Department of Energy

Contract Number DE-AC02-98CH10886 


\section{DISCLAIMER}

This report was prepared as an account of work sponsored by an agency of the United States Government. Neither the United States Government nor any agency thereof, nor any of their employees, nor any of their contractors, subcontractors or their employees, makes any warranty, express or implied, or assumes any legal liability or responsibility for the accuracy, completeness, or any third party's use or the results of such use of any information, apparatus, product, or process disclosed, or represents that its use would not infringe privately owned rights. Reference herein to any specific commercial product, process, or service by trade name, trademark, manufacturer, or otherwise, does not necessarily constitute or imply its endorsement, recommendation, or favoring by the United States Government or any agency thereof or its contractors or subcontractors. The views and opinions of authors expressed herein do not necessarily state or reflect those of the United States Government or any agency thereof. 


\title{
Inellastic X-Ray Scattering at Ultrahigh Pressures
}

\author{
Ho-kwang Mao†, Chichang Kaot and Russell J. Hemley† \\ $\lceil$ Geophysical Laboratory and HPCAT, Carnegie Institution, 5251 Broad Branch Road \\ NW, Washington, DC 20015-1305 \\ \$National Synchrotron Light Source, Brookhaven National Laboratory, Upton, N.Y., \\ 11973
}

\begin{abstract}
Ahstract. Inelastic x-ray scattering (IXS) provides high-pressure research with an arsenal of analytical capabilities for key measurements that were previously unattainable, and high pressure research provides IXS with numerous applications where the technique has unique advantages over other methods. High-pressure investigations can now be conducted using non-resonant IXS, resonant IXS, nuclear resonant IXS, and x-ray emission spectroscopy with energy resolutions of $100 \mathrm{meV}$ to $1 \mathrm{eV}$ for electronic transitions and 1 to $10 \mathrm{meV}$ for phonon studies. By pressure-tuning materials over a wide range, we are able to investigate fundamental physics of electron gases, strongly correlated electron systems, highenergy electronic excitations, and phonons in energy and momentum space. The results will have a profound influence on materials applications as well as providing basic information for understanding the deep interior of the Earth and other planets.
\end{abstract}

\section{Interdisciplinary High Pressure Sciences}

Extreme pressure conditions radically alter the properties of materials. Studies of such states of matter have important implications for a wide range of problems, from chemistry and materials science [1], to fundamental physics [2], to Earth and planetary sciences [3]. Exploration of the pressure variable has revealed new classes of materials. Rare gases, normally classified as chemically inert, combine with other elements under pressure [4-6]. Normally unreactive metals also relinquish their inertness to form new alloys with other elements [7]. Silicon-oxygen tetrahedral frameworks, the basis of rockforming minerals, are destroyed and replaced by altogether different structures at pressures equivalent to those found decp within the Earth [8]. Carbon forms tetrahedral frameworks, and carbon dioxide crystallizes into a quartz-like superhard material $[9,10]$. Carbon rings, basic structural units of organic chemicals and biomaterials, become unstable and are replaced by diamond-like structures. Since most of the matter in the solar system exists under extreme pressures, the flurry of new findings is changing our understanding of the nature and evolution of the planets [11,12]. New non-crystalline states appear [13,14], high-temperature superconductivity is enhanced [15], insulating solids undergo novel transitions to metals [16] and superconductors [17], and there is the prospect for observing entirely new physical phenomena. 


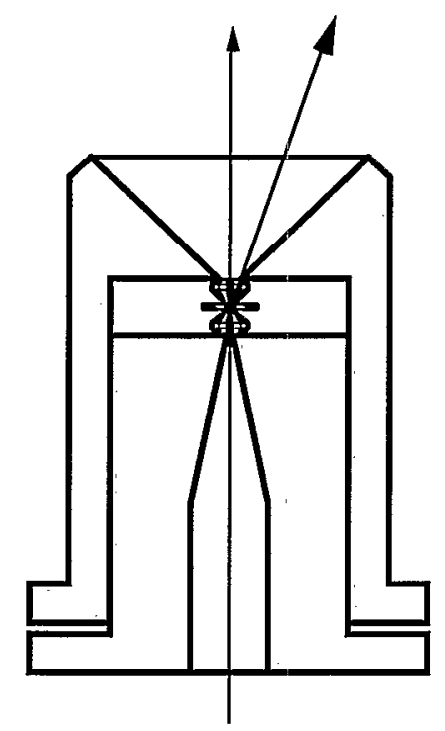

(A)

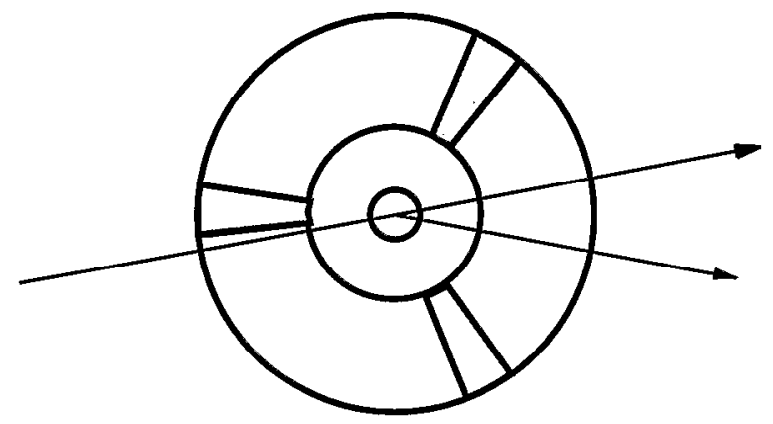

(C)
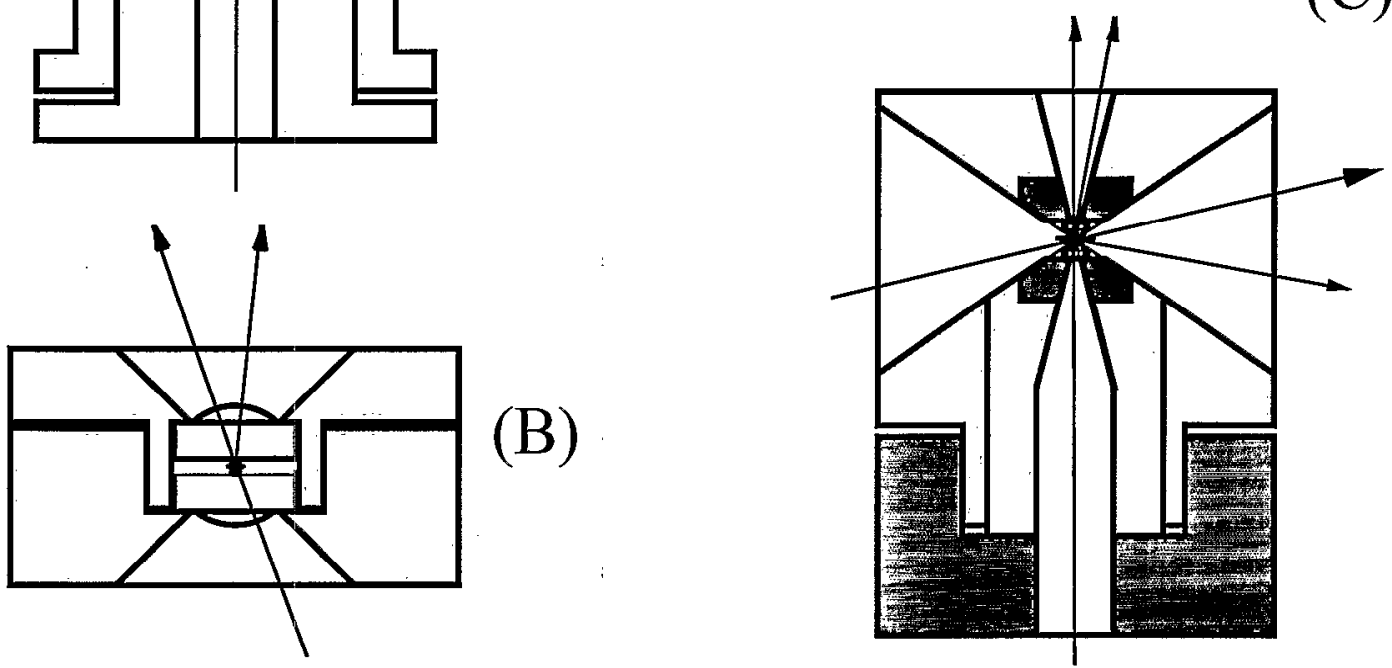

Fig. 1. X-ray scattering geometry in various types of diamond cells. (A) Scattering through narrow openings of the diamond cell axis; (B) axial scattering through Be seats; (C) panoramic scattering through $\mathrm{Be}$ gasket and axial scattering.

What has made this new field of research possible is the sustained development of a broad range of high-pressure techniques based on the diamond cell. Numerous types of cells are in use but all consist of a pair of gem diamonds that pressurize the sample contained inside a small chamber formed within a metal shim, or gasket, placed between the tips of the anvils. Invented over 35 years ago [18], this device has undergone several generations of evolution, especially during the last decade. Now, sample temperatures in excess of those at the surface of the sun $(\mathrm{T}>7000 \mathrm{~K}$ ) (see review [19]) as well as ultra low temperatures down to ten millikelvin $[20,21]$ can be achieved -- while samples are under high pressure. Hand in hand with these developments in pressure generation, there have been extensive advances in research techniques for obtaining accurate measurements of physical and chemical properties of the samples contained within these high-pressure devices. With this concurrent advancement in pressure generation and analytical techniques, the new diamond cell provides a powerful vehicle for reaching extreme pressure conditions, as well as a wide, clear window for comprehensive in-depth 
investigations. For instance, the development of electrical conductivity and magnetic susceptibility [22] microprobes has led to measurement of the record-high superconducting temperature (164 K) [23] and the discoveries of the high-pressure chalcogen superconductors (among which the sulfur becomes a record-high elemental superconductor at $17 \mathrm{~K}$ and $1.5 \mathrm{Mbar}(150 \mathrm{GPa})$ [17]). The developments of laser [24, 25], optical, and infrared spectroscopies [26,27], which probe vibrational, electronic, and magnetic states in molecules and solids at high pressures, has led to the discoveries of quantum and classical orderings of solid hydrogen [28] and symmetrization of ice [29, 30]. The developments of synchrotron $x$-radiation for diffraction, scattering, and spectroscopy has helped to characterize samples under extreme conditions [31-33] and led to discoveries of myriad new phenomena $[34,35]$ and new classes of materials $[4,10$, $14,36]$.

\section{Studies of Material Dynamics by Inelastic X-Ray Scattering at High Pressures}

High-pressure research has only fully utilized the elastic scattering (diffraction) component of the synchrotron source for static structural investigation. At the forefront of synchrotron techniques are those exploiting the enormous potential of inelastic x-ray scattering (IXS) for high-pressure dynamics research. In IXS, an incident x-ray photon with energy $E_{1}$, wave vector $\kappa_{1}$, and polarization $\varepsilon_{1}$, is scattered by the sample and altered into a photon with energy $E_{2}$, wave vector $\kappa_{2}$, and polarization $\varepsilon_{2}$. The energy transfer is $E_{1}-E_{2}$, and the momentum transfer is $4 \pi \sin (\theta / 2) / \lambda$ where $\theta$ is the scattering angle. Experimentally, IXS measures the dynamic structure factor, $S(q, \omega)$, which covers an extremely wide variety of electron and phonon dynamics. With the $\mathrm{x}$-ray wavelength comparable to the scale of the wave vector, dispersion over the entire Brillouin zone can be probed. Complementary to inelastic scattering of light, electrons, and neutrons, IXS is relatively new and is only becoming a realistic experimental tool with the arrival of the extremely brilliant, high-energy synchrotron sources. For materials at ambient conditions, the most basic properties suitable for IXS studies have been determined decades ago by other methods. The frontier of IXS research has shifted to a number of "exotic" properties (e.g., phonons at mesoscopic wavelengths [37,38]). At megabar pressures, however, other methods are often rendered unusable or very restrictive. Techniques employing electron, ultraviolet, or soft x-ray beam require a high-vacuum environment, in direct contradiction to the high-pressure environment in which the pressure vessel totally blocks the beam. Inelastic neutron scattering requires $\mathrm{mm}$ to $\mathrm{cm}$ samples that are limited to pressures below $20 \mathrm{GPa}$ [39]. Consequently, even the most fundamental properties of the simplest materials, such as the electronic band structure of silicon or phonon dispersion of iron or ice, could not be measured at high pressure until very recently. Free of these limitations, IXS holds the promise of fulfilling the technological development essential to the full exploration of the interdisciplinary highpressure sciences. 


\section{High Energy Electronic Excitations at High Pressure}

The stationary states of the fundamental Schrödinger equation for electrons are clearly functions of volume and hence altcrablc by pressure. Pressure has drastic effects on the energy and dispersion of all electronic bands. Such effects are manifested as bands that cross the Fermi surface causing pressure-induced insulator-to-metal transitions.

However, observations in the past have been limited to a narrow energy window. Many electronic levels, including some of the most intriguing pressure-induced changes, occur outside the intrinsic band gap ( $5 \mathrm{eV}$ ) of the diamond window, and are thus inaccessible by conventional optical probes. For example, for the vast range of compression of hydrogen from a molecular solid to proposed metallic states, the density increases by more than a factor of ten $[33,40]$. A metallic conductive state has been observed in hightemperature fluid hydrogen [41]. In low-temperature solid hydrogen the electronic states are predicted to change from initially free molecular-like levels, to a wide-gap insulator with bound excitons, to indirect band-gap closure, and eventually to an alkali metal-like free electron gas (following structural transitions). The fascinating process has been the focus of a large number of theoretical calculations over the past 70 years [42-46], but the electronic transitions, particularly the band structure and excitons in the insulating state, are largely outside the optical observation window.

Using a $10 \mathrm{keV}$ x-ray beam for excitation, IXS spectroscopy gains unlimited access to probe high energy electronic levels. Schell et al. [47] successfully demonstrated the first measurement of a $21.9 \mathrm{eV}$ electronic excitation in solid helium at $61.5 \mathrm{MPa}$ and $4.3 \mathrm{~K}$. Several major experimental challenges, however, need to be overcome before IXS can be applied to megabar pressure samples routinely. First, the double differential scattering cross section of IXS is very small. Second, all the available x-rays need to be focused on to the sample, whose size is on the order of 10-50 microns. Third, for IXS measurements with $100 \mathrm{meV}$ to $1 \mathrm{eV}$ resolution obtained by available $\mathrm{x}$-ray analyzers, the $\mathrm{x}$-ray energy is typically between 8 to $10 \mathrm{keV}$. At these $\mathrm{x}$-ray energies, the absorption of diamond is very high. The first two difficulties have been resolved to a large extent by the recent improvements in both flux and brightness in sources and the development of microfocusing optics. The third difficulty is avoided by the use of a Be gasket (Fig. 2) and a panoramic scattering geometry (Fig. 1) which allows the x-ray beam to enter and exit the diamond anvil cell mainly through the Be gasket $[48,49]$. The combination of these advances enables us to begin IXS experiments using diamond cells. 

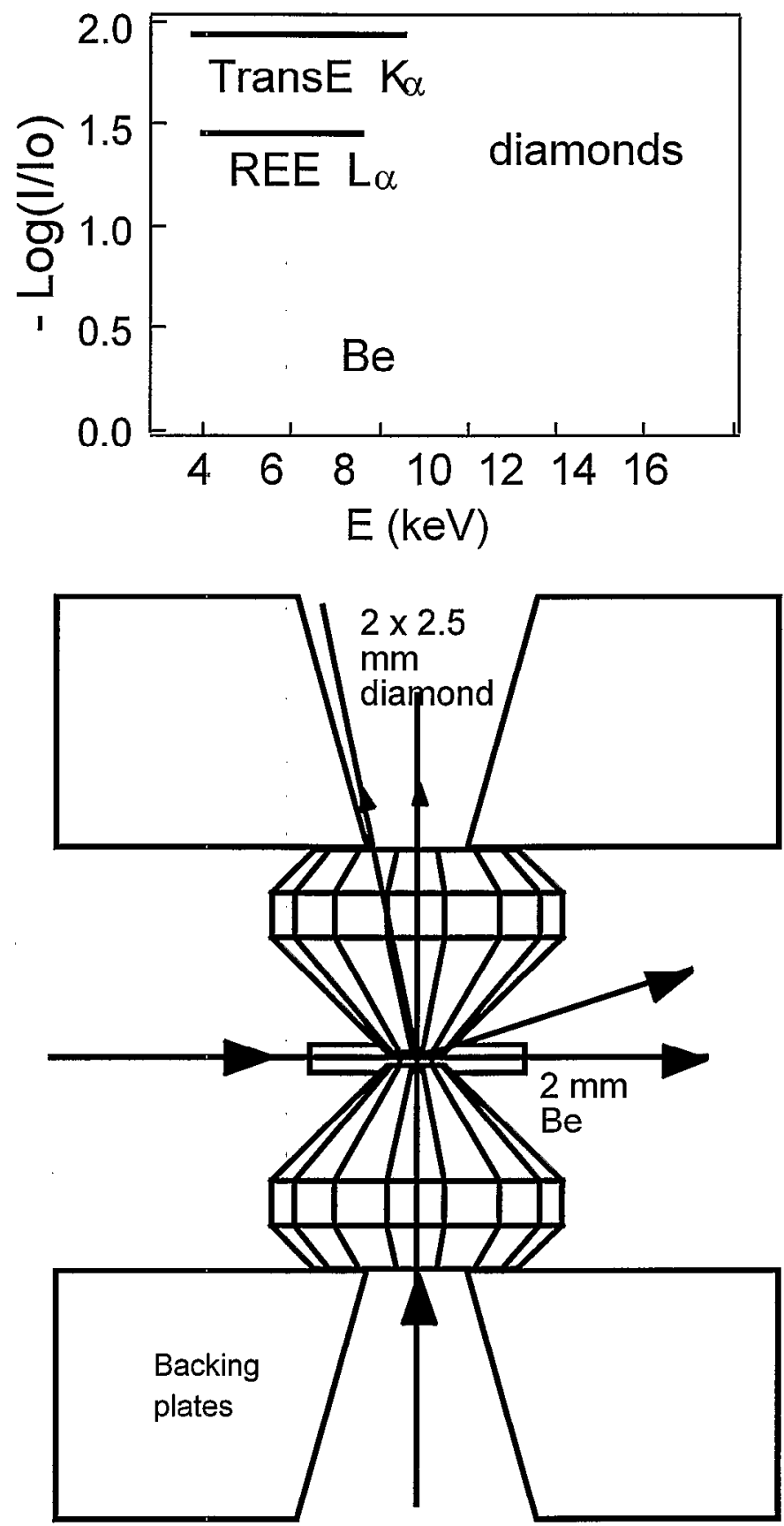

Fig. 2. Absorption of $\mathrm{x}$-ray in the axial geometry through the diamonds and radial geometry through the Be gasket.

\section{Compression of Electron Gas in "Simple Metals"}

The electron gas of simple metals is one of the oldest and most studied many-body problems in condensed-matter physics. However, the dynamic structure factor $S(q, \omega)$ of 
a three-dimensional electron gas is well defined only for a hypothetical metallic density $r_{s}$ $<1$ where the random phase approximation (RPA) applies and for $r_{s}>20$ where the "Wigner lattice" ordering occurs [50]. For real metals in which $r_{s}$ typical falls between 2 and 6 , the lattice effects arc important, and the RPA must be modified by a controversial local field correction factor $g(\boldsymbol{q})$. IXS has proven to be the ideal tool for experimentally studying the full dynamic response function of the electron gas and has been applied to simple metals including $\mathrm{Li}, \mathrm{Na}, \mathrm{Al}$, and $\mathrm{Be}$ [51-57]. Each metal has a discrete $r_{s}$ value at ambient pressure. Comparison of the electron gas in these systems is also partially affected by their different core electrons. To garner fundamental understanding of the electron gas, pressure provides a variable for continuously tuning the band structure of real metals over a wide range of $r_{s}$, thus showing the overall picture of the electron gas at different densities.

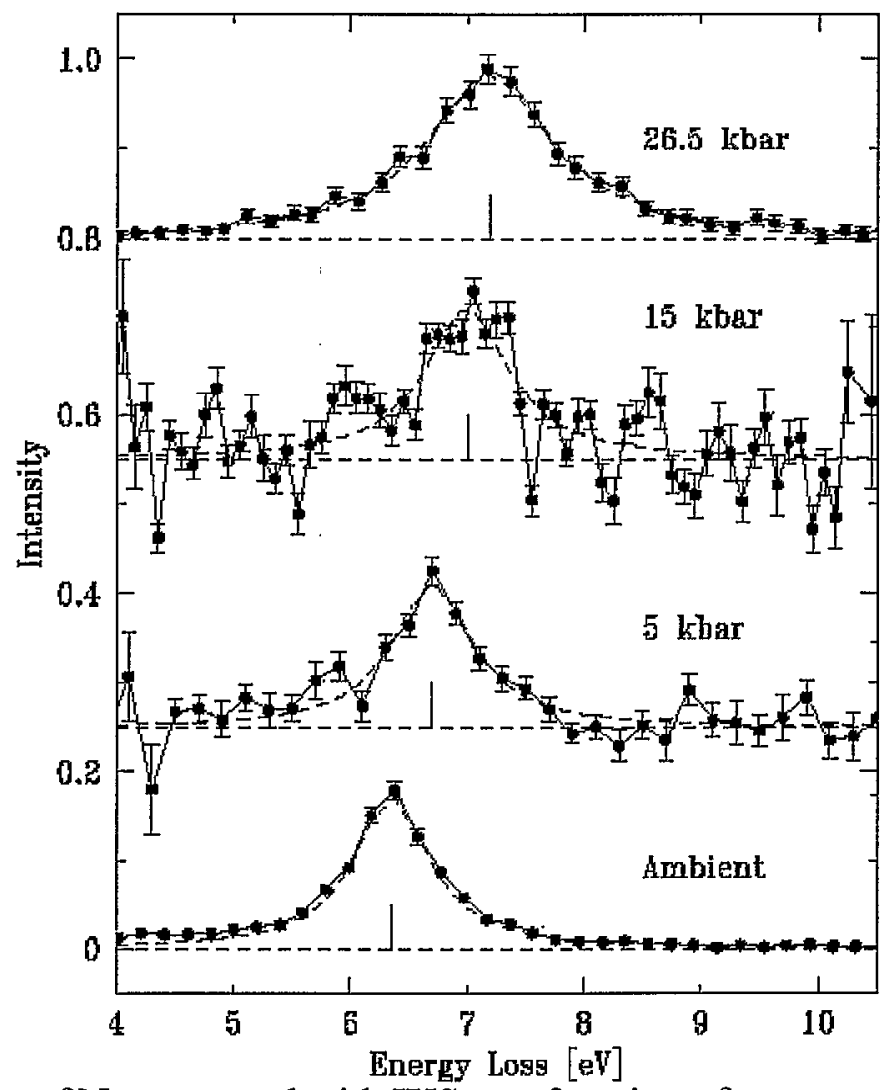

Figure 3. Plasmon of Na measured with IXS as a function of pressure in a diamond cell.

Recently we have performed the IXS study of collective excitations of valence electrons in a diamond cell. Specifically, the plasmon energy of Na was measured as a function of density or pressure. As the pressure increased from ambient to $26.5 \mathrm{kbar}(2.65 \mathrm{GPa})$, the plasmon energy of Na increased from $6.35 \mathrm{eV}$ to $7.19 \mathrm{eV}$. The sample size was about $0.5 \mathrm{~mm}$ (diameter) $\mathrm{x} 0.1 \mathrm{~mm}$ (thickness), the incident x-ray energy used was $10 \mathrm{keV}$, and the scattering angle was $6^{\circ}$. Measurements up to $15 \mathrm{kbar}$ were carried out at X21 hybrid 
wiggler beamline at the National Synchrotron Light Source (NSLS), and the measurement at $26.5 \mathrm{kbar}$ was performed at 13-ID of GeoSoilEnviro Consortium for Advanced Radiation Sources (GSECARS) of the Advanced Photon Source (APS). In both cases the incident photon flux of about $5 \times 10^{10} \mathrm{photon} / \mathrm{sec}$ was measured, and the total energy resolution of the experiment was about $1 \mathrm{eV}$. The difference in data quality is due to a set of micro-focusing Kirkpatrick-Baez (K-B) mirrors [58] that were used at GSECARS to reduce the beam size to $10 \mu \mathrm{m} \times 10 \mu \mathrm{m}$, thus completely discriminating the $\mathrm{Be}$ background. Fig. 3 shows the measured inelastic $\mathrm{x}$-ray scattering spectra of $\mathrm{Na}$. The background due to inelastic scattering from the Be gasket has been removed by fitting the raw data with a single Lorentzian and a third order polynomial background. The dashed lines in Fig. 3 show the best-fit Lorentzian lineshape using fitted parameters. The measured values agreed well with the RPA calculation.

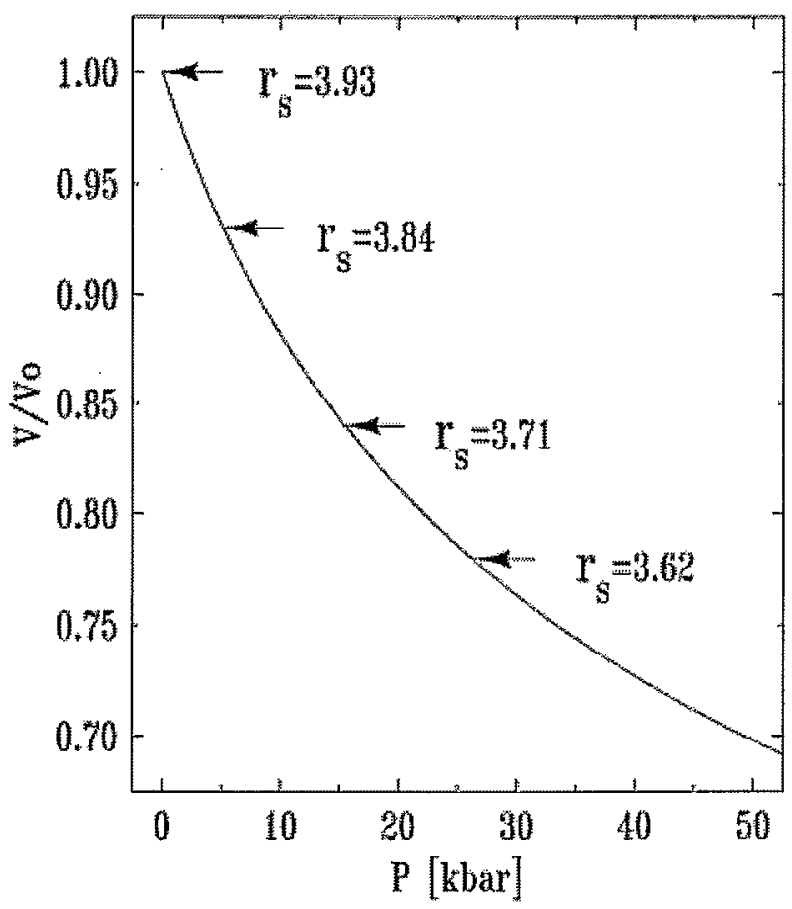

Figure 4. Pressure - volume $\left(r_{s}\right)$ relation of $\mathrm{Na}$ at ambient temperature.

The experiment demonstrates the power of pressure to tune various properties as well as the advantages of integrated studies. For instance, Hong and Lee [59] derived an analytic expression for the dynamic structure factor $S(q, \omega)$ for the electron gas. The asymptotically exact solution is only valid at one particular electron density of $r_{s}=3.5$, but no real metal at ambient conditions has this density. Lithium $\left(r_{s}=3.25\right)$ is the closest for comparison [51], and $\mathrm{Na}\left(r_{s}=3.93\right)$ is significantly higher. Fig. 4 shows the equation of state of $\mathrm{Na}$ determined by $\mathrm{x}$-ray diffraction at ambient temperature and the large range 
of electron densities $\left(r_{s}\right)$ of the experiment. With slightly higher pressures of $45 \mathrm{kbar}$, we will be able to test the predicted singular point at $r_{s}=3.5$.

\section{Dynamic Response of Strongly Correlated Elcetronic Systems}

Strongly correlated electronic systems display rich phenomena ranging from magnetic ordering to superconductivity. As a result, they have attracted particular attention in condensed matter physics. Competition among the Mott-Hubbard interaction energy $U$, the ligand field energy $\Delta$, the $3 d$ bandwidth $W$, and the ligand-metal hybridization interaction $T$ lead to complex electronic, magnetic, and phonon behavior which is strongly manifested in the pressure dimension. Understanding electron correlation effects in strongly correlated electronic systems remains one of the major challenges in solid state physics. In one limit, semi-empirical model Hamiltonians, which can be solved analytically or numerically, are constructed to study these systems [60]. In the other limit, first-principles electronic structure calculations are being extended into these systems by introducing new theoretical schemes to include electron correlations [61]. Megabar pressures can be used to modify the high energy scale properties, such as the on-site Coulomb energy, charge transfer energy, and crystal-field splitting of these systems, as evidenced by the large number of phase transitions induced by pressure. Combining IXS with megabar pressure thus provides a unique opportunity to systematically study the effect of these high energy scale parameters in combination with theory, and to study the origin of the large number of phase transitions and the associated changes in their electronic structures.

It has also been found that for narrow-band solids, such as transition-metal and rare-earth compounds, resonant inelastic x-ray scattering can be used to probe specific elementary excitations $[62,63]$. In these experiments on highly correlated electron systems, the incident $\mathrm{x}$-ray energy is tuned through an absorption edge of the sample to exploit the resonant condition in the scattering $[64,65]$. For example, large resonant enhancement of charge transfer excitations was observed in $\mathrm{NiO}$ [66]. A configuration-interaction cluster model of $\mathrm{NiO}$ is used to interpret the scattering cross section and the strong incident energy dependence. This interpretation has been confirmed by the observation of charge transfer excitations in several high-Tc related compounds [67,68]. Recently, polarization and momentum dependent measurements on these compounds have also been reported $[69,70]$.

There have also been a growing number of resonant inelastic scattering studies of core excitations [71-74], i.e. the final states of the inelastic scattering process in these studies are localized shallow core excitations. It should be noted that these shallow core excitations are the same excitations routinely probed by soft $\mathrm{x}$-ray absorption spectroscopy, and are rich in multiplet structures for these highly correlated electronic systems. In fact, the high energy scale parameters of the highly correlated systems are routinely derived from fitting these spectra with model calculations. For magnetic 
samples, excitation with circularly polarized x-rays can also provide information on spinresolved electronic structure [75-77].

We have also explored high-resolution x-ray emission spectroscopy (HRXES) [78] for the study of strongly correlated electronic systems at high pressure. In HRXES, deep-core electrons in the sample are excited by x-rays. The core-holes then decay through radiative processes and produce fluorescent photons. The energies of the fluorescent photons are analyzed with sub-eV energy resolution of the emission spectral lineshape to provide information on the filled electronic states of the sample. The final state of the fluorescent process is a one-hole state, identical to the final state of the photoemission process. Photoelectron spectroscopy is an important tool for studying large chemical shifts in the core-level binding energies and the valence band density of states, but requires a vacuum environment incompatible with high pressures. As an alternative tool, HRXES solves the problem for in-situ measurements at high pressure.

In HRXES, the incident beam is unspecified; x-rays of sufficiently high energy for penetrating the pressure vessel can be used to excite the emission. Only the element specific emission x-rays must exit the vessel, reducing half of the absorption problem. With the low end of the high-pressure energy window extended down to $4 \mathrm{keV}$ by the high-strength beryllium gasket [48], all elements above $\mathrm{Ca}(\mathrm{Z}=20)$ can be studied at high pressures by a suitable choice of analyzer crystals. In particular, the important $3 d$ and $4 f$ emissions will now be open for high-pressure research. This covers the transition elements and rare-earth elements with specific electric, magnetic, and optical properties crucial for a wide range of applications in materials sciences and technology. For instance, transition-element ions, with their variable valence and magnetic states, control major geochemical processes and geophysical behaviors, such as oxidation, reduction, chemical differentiation, elasticity, geomagnetism, conductivity, and radiation heat transfer. Magnetic collapse in transition-metal monoxides has been predicted from firstprinciples computations at pressures of the lower mantle and core [79]. We have studied high-pressure HRIXS of iron oxides and sulfides at the NSLS and APS. High-resolution $\mathrm{K}_{\beta}$ emission of ferrous ion in troilite shows a high-spin to low-spin transition at $4 \mathrm{GPa}$ coinciding with the FeS I-II structural transition [80]. Likewise, a high-spin to low-spin transition occurs in hematite at $50 \mathrm{GPa}$. Wüstite, on the other hand, remains high spin to pressures as high as $140 \mathrm{GPa}$ [81]. High-resolution $\mathrm{K}_{\gamma}$ emission spectra of these minerals reveal their oxygen bonding characteristics. The results place important constraints on models of the deep mantle and cores of terrestrial planets.

\section{Phonon Vibrational Dynamics at High Pressures}

The effect of pressure on phonon dynamics reveals a wealth of information on fundamental interatomic interactions, propagation of elastic waves, mechanical stability of solids, phase transition mechanisms, vibrational energies, and thermodynamic

parameters. Raman and infrared spectroscopies have been used extensively for high- 
pressure studies of dynamics (for example see [82-84]), but momentum resolution is not straightforward partly because the stressed diamond anvils changes the polarization of the probing light beam. Complete $(q \neq 0)$ phonon studies are normally carried out by inelastic neutron scattering (INS), which requires centimeter-size samples currently impossible for ultrahigh pressure research. A recent phonon dispersion study of singlecrystal diamond has demonstrated that the resolution of IXS could be on a par with INS in its coverage of full momentum space [85]. With the optimized size of the focused $x-$ ray beam and sample, similar quality of data can be obtained on single-crystal samples at high pressures to 50-100 GPa. This opens tremendous opportunities for complete characterization and tuning phonon dynamics over a large range of density.
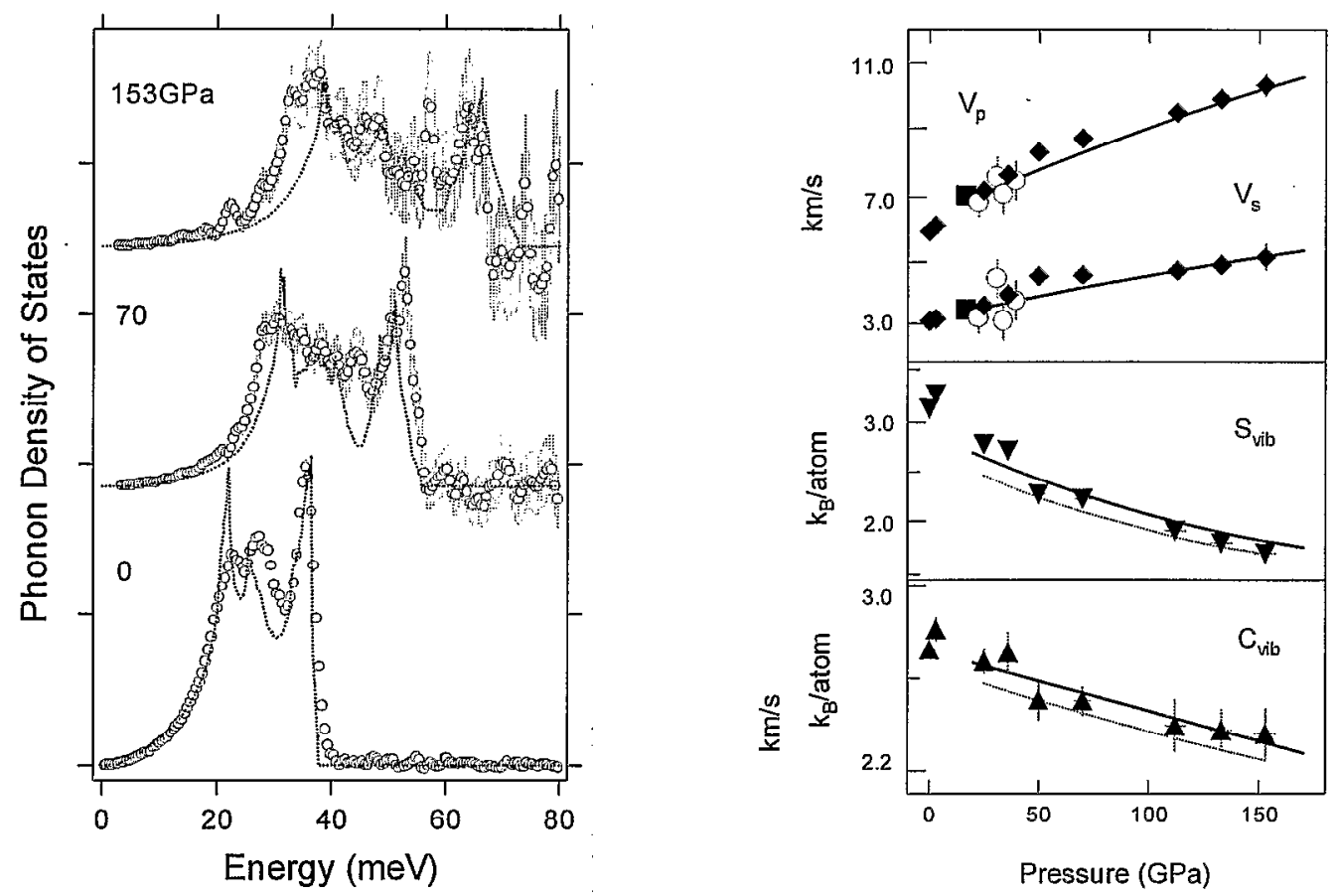

Fig. 5. Left: Phonon DOS of iron. Right: longitudinal velocity $\left(V_{P}\right)$, shear velocities $\left(V_{S}\right)$, vibrational entropy $\left(S_{v i b}\right)$, and vibrational heat capacity $\left(C_{v i b}\right)$ of iron as a function of pressure. Symbols, error bars, and solid curves are the NRIXS experimental results; thin dashed curves are from $a b$ initio theory [86]. 


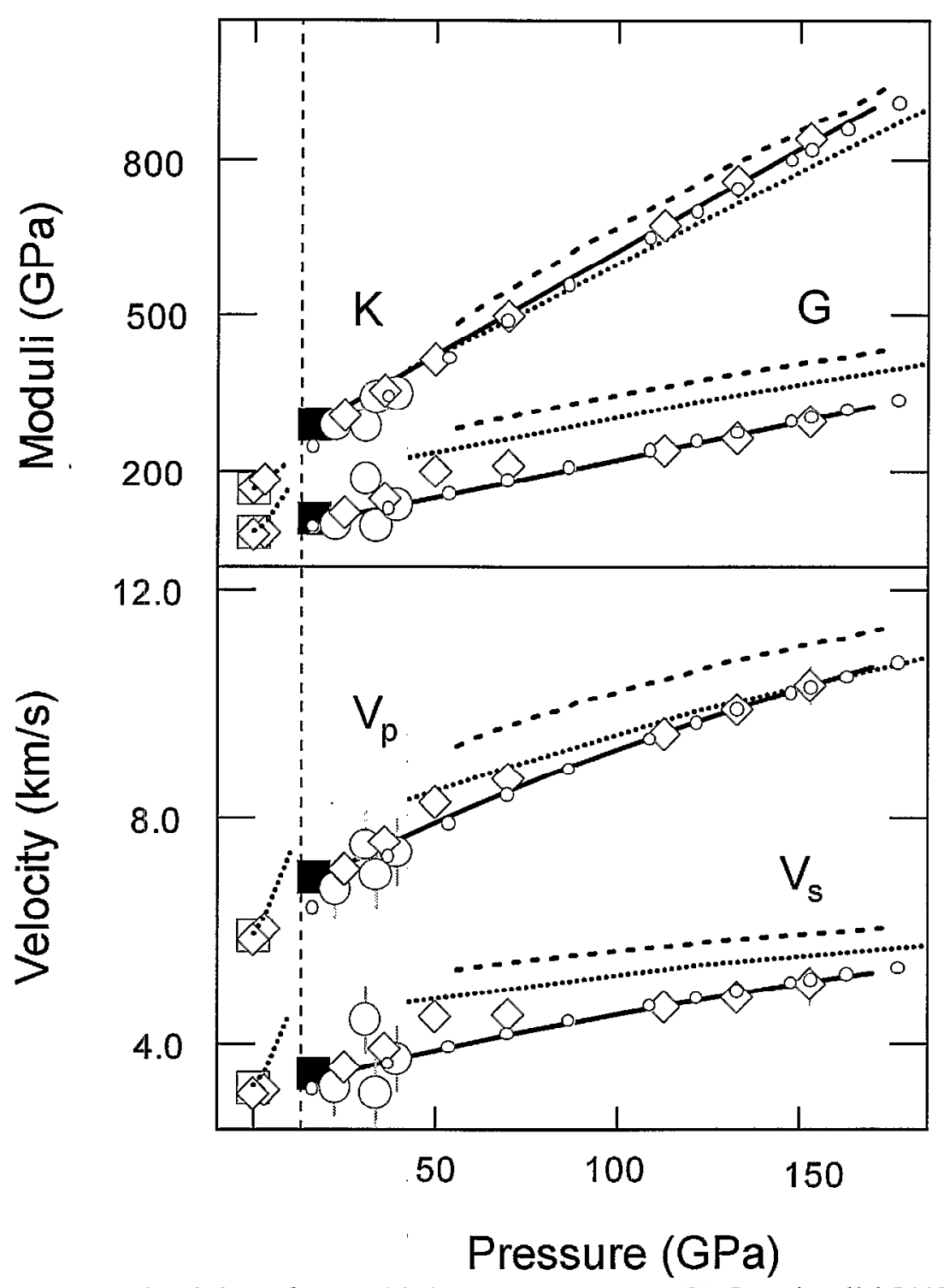

Fig 6. Aggregate elasticity of $\mathrm{Fe}$ at high pressures; open [87] and solid [49] squares, ultrasonic data; circles, RXD data with Au stress calibration [49]; small circles, RXD extrapolation based on constant $K / G$ [49]; diamonds, present NRIXS results; solid curves, curve-fitting through the ultrasonic and the NRIXS data; dotted curve, calculated from the slope at the gamma point of the present $a b$-initio theoretical phonon dispersion curves; dashed curve, ab-initio calculations of Steinle-Neumann et al. [88]. The vertical line at $13 \mathrm{GPa}$ separates bcc Fe and high-pressure hop Fe.

Phonon densities of state (DOS) can be obtained by IXS measurement of polycrystalline samples integrated through the entire Brillouin zone. The acoustic velocity is directly derived from the dispersion of the acoustic phonon energy [89]. Studies of phonon DOS can be greatly simplified if the element involved has a nuclear resonant Mössbauer effect. 
For instance, the nuclear resonant inelastic x-ray scattering (NRIXS) method developed for the Mössbauer isotope ${ }^{57} \mathrm{Fe}$ has produced DOS measurements of bcc-Fe at ambient conditions identical to the INS results [90]. We successfully extended the NRIXS technique to high pressure and obtained the DOS of hcp-Fe up to $153 \mathrm{GPa}$ [91]. The high-pressure vibrational dynamics of iron has attracted particular attention among physicists and geophysicists because iron is an archetypal transition element $[92,93]$ and is a dominant component in the cores of the Earth and other terrestrial planets (Mars, Venus, Mercury). Many important dynamic, thermodynamic, and elastic properties, including vibrational kinetic energy $\left(E_{k}\right)$, zero-point vibrational energy $\left(E_{Z}\right)$, vibrational entropy $\left(S_{v i b}\right)$, and vibrational heat capacity $\left(C_{v i b}\right)$, Debye temperature $\left(\Theta_{D}\right)$, Grüneisen parameter $(\gamma)$, thermal expansivity $(\alpha)$, longitudinal velocity $\left(V_{P}\right)$, shear velocities $\left(V_{S}\right)$, bulk modulus $(K)$, and shear modulus $(G)$ are derived from the phonon DOS (Fig. 3 and 4). These properties are essential for interpreting seismological observations [94, 95] and numerical modeling of planetary cores, but have previously eluded direct experimental study and have been dependent solely upon theoretical calculations.

\section{Conclusions}

The handful of examples demonstrate the potential of the IXS as a powerful tool for probing materials dynamics at high pressures. With the full integration of the high pressure and the IXS instrument, we expect an order of magnitude improvement in the signal-to-background ratio. In addition, with the recent development of synchrotron sources, we expect to have $2 \times 10^{13}$ photons/sec within $1 \mathrm{eV}$ bandwidth. The more than 2 orders of magnitude gain in flux and one order in sampling discrimination can be used for ultrahigh-pressure studies of smaller samples or higher $Z$ elements with high energy resolutions. Fundamental behavior of the electron gases, strongly correlated electronic systems, and phonon dynamics can be investigated over an extended range of $30-300$ $\mathrm{GPa}$. In addition to the importance to basic physics and materials sciences, knowledge of high-pressure behavior is important for exploring of the properties of Earth and planetary interiors.

\section{Acknowledgments}

The recent experiments described above were performed with the help of J. Shu and J. Hu. This work was supported by the NSF, NASA, DOE and W. M. Keck Foundation. We thank NSLS and APS for providing synchrotron radiations.

[1] McMillan P F 1998 Nature 391539

[2] Hemley R J and Ashcroft N W 1998 Physics Today 5126

[3] Hemley R J 1999 Science 2851026

[4] Vos W L, Finger L W, IIemley R J, IIu J, Mao II K and Schouten J A 1992 Nature 35846 
[5] Loubeyre P, Jean-Louis M, Toullec R L and Charon-Gerard L 1993 Phys. Rev. Lett. 70178

[6] Loubeyre P, Letoullec R and Pinceaux J P 1994 Phys. Rev. Lett. 721360

[7] Parker L J, Atou T and Badding J V 1996 Science 27395

[8] Knittle E and Jeanloz R 1987 Science 235668

[9] Iota V, Yoo C S and Cynn H 1999 Science 2831510

[10] Yoo C S, Cynn H, Gygi F, Galli G, Iota V, Nicol M, Carlson S, Häusermann D and Mailhiot C 1999 Phys. Rev. Lett. 835527

[11] Duffy T S, Vos W L, Zha C-S, Hemley R J and Mao H K 1994 Science 2631590

[12] Fei Y, Prewitt C T, Mao H K and Bertka C M 1995 Science 2681892

[13] Mishima O, Calvert L D and Whalley E 1984 Nature 310393

[14] Hemley R J, Jephcoat A P, Mao H K, Ming L C and Manghnani M 1988 Nature 334 52

[15] Gao L, Xue Y Y, Chen F, Xiong Q, Meng R L, Ramirez D, Chu C W, Eggert J H and Mao H K 1994 Physica C 1493

[16] Weir S T, Mitchell A C and Nellis W J 1996 Phys. Rev. Lett. 761860

[17] Struzhkin V V, Hemley R J, Mao H K and Timofeev Y A 1997 Nature 390382

[18] Weir C E, Lippincott E R, VanValkenburg A and Bunting E N 1959 J. Res. NBS 63A 55

[19] Shen G and Heinz D L 1998 Ultrahigh-Pressure Mineralogy: Physics and Chemistry of the Earth's Deep Interior ed Hemley R J (Washington, D. C.: Mineralogical Society of America) p 369

[20] Haselwimmer R K W, Tyer A W and Pugh E 1998 Proceedings of International Conference- AIRAPT-16 and HPCJ-38- on high pressure science and technology ed Nakahara M (Kyoto: Japan Society of High Pressure Science and Technology) p 481

[21] Eremets M I, Gregoryanz E, Struzhkin V V, Mao H K, Hemley R J, Moulders N and Zimmerman N M 2000 Phys. Rev. Lett. 852797

[22] Timofeev Y A, Mao H K, Struzhkin V V and Hemley R J 1999 Rev. Sci. Instrum. 70 4059

[23] Gao L, Xue Y Y, Chen F, Xiong Q, Meng R L, Ramirez D, Chu C W, Eggert J H and Mao H K 1994 Phys. Rev. B, Rapid Comm. 504260

[24] Hemley R J and Mao H K 1988 Phys. Rev. Lett. 61857

[25] Hemley R J and Mao H K 1989 Ann. Rep. Geophys. Lab. 79

[26] Hanfland M, Hemley R J, Mao H K and Williams G P 1992 Phys. Rev. Lett. 691129

[27] Hanfland M, Hemley R J and Mao H K 1993 Phys. Rev. Lett. 703760

[28] Mazin I I, Hemley R J, Goncharov A F, Hanfland M and Mao H K 1997 Phys. Rev. Lett. 781066

[29] Goncharov A F, Struzhkin V V, Somayazulu M, Hemley R J and Mao H K 1996 Science 273218

[30] Struzhkin V V, Goncharov A F, Hemley R J and Mao H K 1997 Phys. Rev. Lett. 78 4446

[31] Mao H K, Wu Y, Hemley R J, Chen L C, Shu J F, Finger L W and Cox D L 1990 Phys. Rev. Lett. 641749 
[32] Meade C, Hemley R J and Mao H K 1992 Phys. Rev. Lett. 691387

[33] Loubeyre P, LeToullec R, Hausermann D, Hanfland M, Hemley R J, Mao H K and Finger L W 1996 Nature 383702

[34] Hemley R J, Soos Z G, Hanfland M and Mao H K 1994 Nature 369384

[35] Loubeyre P, LeToullec R, Wolanin E, Hanfland M and Hausermann D 1999 Nature 397503

[36] Vos W L, Finger L W, Hemley R J and Mao H K 1996 Chem. Phys. Lett. 257524

[37] Ruocco G, Sette F, Bergmann U, Krisch M, Masciovecchio C, Mazzacurati V, Signorelli G and Verbeni 1996 Nature 379521

[38] Masciovecchio C, Monaco G, Ruocco G, Sette F, Cunsolo A, Krisch M, Mermet A, Soltwisch M and Verbeni R 1998 Phys. Rev. Lett. 80544

[39] Klotz S and Braden M 2000 Phys. Rev. Lett. 853209

[40] Mao H K and Hemley R J 1994 Rev. Mod. Phys. 66671

[41] Nellis W J, Weir S T and Mitchell A C 1999 Phys. Rev. B 593434

[42] Kohanoff J, Scandolo S, Gironcoli S d and Tosatti E 1999 Phys. Rev. Lett. 834097

[43] Nagao K and Nagara H 1998 Phys. Rev. Lett. 80548

[44] Lenosky T J, Kress J D, Collins L A and Kwon I 1997 Phys. Rev. B 55 R11

[45] Ashcroft N W 1968 Phys. Rev. Lett. 211748

[46] Wigner E and Huntington H B 1935 J. Chem. Phys. 3764

[47] Schell N, Simmons R O, Kaprolat A, Schülke W and Burkel E 1995 Phys. Rev. Lett. 742535

[48] Hemley R J, Mao H K, Shen G, Badro J, Gillet P, Hanfland M and Häusermann D 1997 Science 2761242

[49] Mao H K, Shu J, Shen G, Hemley R J, Li B and Singh A K 1998 Nature 396741

[50] Platzman P M and Eisenberger P 1974 Phys. Rev. Lett. 33152

[51] Eisenberger P, Platzman P M and Schmidt P 1975 Phys. Rev. Lett. 3418

[52] Schulke W, Nagasawa H and Mourikis S 1984 Phys. Rev. Lett. 522065

[53] Hill J P, Kao C C, Caliebe W A C, Gibbs D and Hastings J B 1996 Phys. Rev. Lett. 773665

[54] Platzman P M, Isaacs E D, Williams H, Zschack P and Ice G E 1992 Phys. Rev. B 46 12

[55] Schulke W, Schulte-Schrepping H and Schmitz J R 1993 Phys. Rev. B 4712

[56] Eisenberger P, Platzman P M and Pandy K C 1973 Phys. Rev. Lett. 31311

[57] Schulke W, Nagasawa H, Mourikis S and Kaprolat A 1989 Phys. Rev. B 4012

[58] Eng P J, Newville M, Rivers M L and Sutton S R 1998 X-Ray Microbeam

Technology and Applications ed McNult I Proc. SPIE-Int. Soc. Opt. Eng) p 145

[59] Hong J and Lee M H 1993 Phys. Rev. Lett. 701972

[60] Zaanen J, Sawatzky G A and Allen J W 1985 Phys. Rev. Lett. 55418

[61] Eguiluz A G, Ku W and Sullivan J M 1998

[62] Butorin S M, Mancini D C, Guo J-H, Wassdahl N, Norgren J, Nakazawa M, Tanaka S, Uozumi T, Ma Y, Miyano K E, Karlin B A and Shuh D K 1996 Phys. Rev. Lett. 77574

[63] Kuiper P, Guo J-H, Sathe C, Duda L-C, Norgren J, Pothuizen J J M, Groot F M F d and Sawatzky G A 1998 Phys. Rev. Lett. 805204 
[64] Blume M 1985 J. Appl. Phys. 573615

[65] Hannon J P, Trammell G T, Blume M and Gibbs D 1988 Phys. Rev. Lett. 611245

[66] Kao C-C, Caliebe W A, Hastings J B and Gillet J-M 1996 Phys. Rev. B 5416361

[67] Hill J P, Kao C-C, Caliebe W A, Matsubara M, Kotani A, Peng J L and Grecne R L 1998 Phys. Rev. Lett. 804967

[68] Abbamonte P A, Burns C, Isaacs E D, Platzman P M, Miller L L, Cheong S W and Klein M V 1999 Phys. Rev. Lett. 83860

[69] Enkisch H, Kaprolat A, Schulke W, Krisch M H and Lorenzen M 1999 Phys. Rev. B B60 8624

[70] Hämäläinen K, Hill J P, Huotari S, Kao C-C, Berman L E, Kotani A, Ide T, Peng J L and Greene R L 2000 Phys. Rev. accepted

[71] Ice G E, Isaacs E and Zschack P 1993 Phys. Rev. B 476241

[72] Krisch M H, Kao C-C, Selte F, Caliebe W A, Hamalainen K and Hastings J B 1995 Phys. Rev. Lett. 744931

[73] Bartolome F, Tonnerre J M, Seve L, Raoux D, Chaboy J, Garcia L M, Krisch M and Kao C-C 1997 Phys. Rev. Lett. 793775

[74].Caliebe W A, Kao C-C, Hastings J B, Taguchi M, Kotani A, Uozumi T and Groot F MF d 1998 Phys. Rev. B B58 13452

[75] Caliebe W A, Kao C-C, Berman L E, Hastings J B, Krisch M H, Sette F and Hamalainen K 1996 J. Appl. Phys. 796509

[76] Krisch M H, Sette F, Bergmann U, Masciovecchio C, Verbeni R, Goulon J, Caliebe W A and Kao C-C 1996 Phys. Rev. B 54 R12673

[77] DeGroot F M F, Nakazawa M, Kotani A, Krisch M H and Sette F 1997 Phys. Rev. B B56 7285

[78] Hämäläinen K, Kao C C, Hastings J B, Siddons D P, Berman L E, Stojanoff V and Cramer S P 1992 Phys. Rev. B 4614

[79] Cohen R E, Mazin I I and Isaak D E 1997 Science 275654

[80] Rueff J-P, Kao C C, Struzhkin V V, Badro J, Shu J, Hemley R J and Mao H K 1999 Phys. Rev. Lett. 823284

[81] Badro J, Struzhkin V V, Shu J, Hemley R J, Mao H K, Kao C C, Rueff J-P and Shen G 1999 Phys. Rev. Lett. 834101

[82] Eggert J H, Mao H K and Hemley R J 1993 Phys. Rev. Lett. 702301

[83] Goncharov A F, Hemley R J, Mao H K and Shu J F 1998 Phys. Rev. Lett. 80101

[84] Gorelli F A, Ulivi L, Santoro M and Bini R 1999 Phys. Rev. Lett. 834093

[85] Schwoerer-Böhning M and Macrander A T 1998 Phys. Rev. Lett. 805572

[86] Vocadlo L, Brodholt J, Alfé D and Price G D 1999 Geophys. Res. Lett. 261231

[87] Guinan M W and Beshers D N 1968 J. Phys. Chem. Solids 29541

[88] Steinle-Neumann G, Stixrude L and Cohen R E 1999 Phys. Rev. B 60791

[89] Fiquet G, Badro J, Guyot F, Requardt H and Kirsch M 2000 Science 291468

[90] Sturhahn W, Toellner T S, Alp E E, Zhang X W, Ando M, Yoda Y, Kikuta S, Seto M, Kimball C W and Dabrowski B 1995 Phys. Rev. Lett. 743832

[91] Mao H K, Xu J, Struzhkin V V, Shu J, Hemley R J, Sturhahn W, Hu M, Alp E, Vocadlo L, Alfè D, Price G D, Gillan M J, Schwoerer-Böhning M, Häuscrmann D, 
Eng P, Shen G, Giefers H, Lübbers R and Wortmann G 2001 Science 292 May 4, 2001

[92] Stixrude L and Cohen R E 1995 Science 2671972

[93] Söderlind P, Moriarty J A and Wills J M 1996 Phys. Rev. B 5314

[94] Anderson O L 1995 Rev. Geophys. 33429

[95] Stacey F D 1995 Phys. Earth Planet. Inter. 89219 